\title{
Prognostic value of FOXQI in patients with malignant solid tumors: a meta-analysis
}

\author{
This article was published in the following Dove Press journal: \\ Onco Targets and Therapy \\ 23 March 2017 \\ Number of times this article has been viewed
}

\author{
Xiaohai Cui ${ }^{1-3, *}$ \\ Jing Zhang ${ }^{1, *}$ \\ Jiajun $\mathrm{Lv}^{4}$ \\ Yan Yan' \\ Xu Liu' \\ Jizhao Wang' \\ Yi Lv $v^{2,3,5}$ \\ Jia Zhang'
}

'The Second Department of Thoracic Surgery, ${ }^{2}$ Institute of Advanced Surgical Technology and Engineering, The First Affiliated Hospital of Xi'an Jiaotong University, ${ }^{3}$ Shaanxi Provincial Regenerative Medicine and Surgical Engineering Research Center, ${ }^{4}$ Xi'an Jiaotong University Health Science Center, ${ }^{5}$ Department of Hepatobiliary Surgery, The First Affiliated Hospital of Xi'an Jiaotong University, Xi'an, Shaanxi, People's Republic of China

*These authors have contributed equally to this work
Background: Forkhead box Q1 (FOXQ1, also known as HFH1), a member of the forkhead transcription factor family, has been demonstrated to be overexpressed in multiple tumors and is thought to be an indicator of poor clinical outcomes.

Methods: A meta-analysis using qualified relevant literature was performed to evaluate the prognostic significance of FOXQ1 in various malignant solid tumors. A search of electronic databases was conducted in MEDLINE, Embase, and the Cochrane Library to identify relevant studies published from 1966 to July 30, 2016, and the studies were identified by further evaluation. The pooled hazard ratios (HRs) with 95\% confidence intervals (CIs) for analyses were assessed to investigate the association between FOXQ1 expression and overall survival (OS) of patients with malignant solid tumors.

Results: A total of 1,520 patients from six studies (seven cohorts) with multiple malignant solid tumors were included. For OS, high FOXQ1 expression could significantly predict worse outcome with the pooled HR of 1.38 (95\% CI: $1.17-1.59 ; P<0.001)$. The subgroup analysis suggested that the elevated levels of FOXQ1 appear to be associated with worse OS in hepatocellular carcinoma $(\mathrm{HR}=1.34 ; 95 \% \mathrm{CI}: 1.11-1.57 ; P<0.001)$ and other cancers $(\mathrm{HR}=1.62 ; 95 \%$ CI: $1.09-2.14 ; P<0.001)$.

Conclusion: This meta-analysis indicated that the high expression of FOXQ1 is associated with an adverse OS in malignant solid tumors, suggesting that FOXQ1 may be a predictor of poor prognosis for the development of malignant solid tumors.

Keywords: FOXQ1, meta-analysis, cancer, prognosis, overall survival

\section{Introduction}

Forkhead box Q1 (FOXQ1, also known as HFH1) is a member of the forkhead transcription factor family. ${ }^{1}$ FOXQ1 is located at human chromosome $6 \mathrm{p} 25.3$ and consists of 2,319 bp. ${ }^{2}$ It is encoded by an open reading frame of 1,029 bp and produces a functional protein with 403 amino acids. It is expressed in different tissues, including stomach, salivary gland, duodenum, prostate, fetal liver tissue, bladder, and trachea, and plays important roles in development, metabolism, aging, and cancer. ${ }^{3-5}$ Initially, it has been shown to be involved in hair follicle differentiation, gastrulation, and mucin production in mice. ${ }^{6-8}$ Several recent studies demonstrated that FOXQ1 expression increased in many human cancers, including hepatocellular carcinoma (HCC), colorectal cancer, esophageal cancer, breast cancer, and non-small-cell lung cancer, and its expression correlated with metastasis and poor prognosis..$^{9-19}$ Therefore, FOXQ1 is considered as an oncogenic property.

However, the prognostic value of FOXQ1 overexpression across different malignant solid tumors is still unclear. Consequently, we initiated a meta-analysis to investigate the significance of increased FOXQ1 expression in the prediction of clinical outcomes of patients with solid tumors. 


\section{Methods}

\section{Search strategy}

A search of electronic databases was conducted in MEDLINE, Embase, and the Cochrane Library to identify relevant studies published from 1966 to July 30, 2016, using the random combination of the following three sets of keywords: "forkhead box Q1 OR FOXQ1 OR HFH1”, “carcinoma OR cancer OR malignancy OR tumor", and "prognosis OR survival OR outcome". In addition, we manually searched references of the selected articles for the potential articles that have been missed at the first search.

\section{Inclusion and exclusion criteria}

The following criteria were applied to include the eligible studies: 1) human-based investigations, 2) must be a pathologically confirmed certain type of solid tumor, 3) articles published in English with full texts, 4) usage of immunohistochemistry (IHC) method to investigate the FOXQ1 expression in the primary tumor tissues, 5) reporting the correlation between FOXQ1 expression and overall survival (OS), and 6) availability of hazard ratios (HRs) and their 95\% confidence intervals (CIs) directly or indirectly by calculating from the original data. The following ineligible studies were excluded: 1) studies conducted using cell lines and animals, 2 ) the types of case reports, letters, reviews, or meta-analyses, 3) studies published in non-English, 4) studies lacking necessary data, and 5) overlapped studies or duplicated data.

\section{Data extraction}

Data were extracted from each study in duplicate by two independent researchers (Xiaohai Cui and Jia Zhang) based on the inclusion and exclusion criteria described earlier. Disagreement was settled by consulting with a third investigator (Jing Zhang). Extracted data from eligible studies included the first author's surname, year of publication, country, histology, number of patients, assay method, positive percentage, the sources of $\mathrm{HR}(95 \% \mathrm{CI})$, and survival results. HRs and $95 \%$ CIs were obtained from the original article in all cases.

\section{Quality assessment}

Newcastle-Ottawa scale (NOS) was used to perform the quality assessment of each study. ${ }^{20}$ Based on three items, including election, comparability, and outcome, the total score of NOS ranged from 0 to 9 . A score of $>6$ is considered as high quality.

\section{Statistical analysis}

Chi-square test-based Cochran's Q and $I^{2}$ statistics were used to calculate the heterogeneity of the individual HR.
For Q statistics, $P<0.05$ was considered as statistically significant. For $I^{2}$ statistics, $I^{2}<25 \%$ means no heterogeneity, $25 \%<I^{2}<50 \%$ means moderate heterogeneity, and $I^{2}>50 \%$ means strong heterogeneity. If no obvious heterogeneity was found among studies, the fixed effects model was used to combine the individual HR estimates; otherwise, the random effects model was applied. For FOXQ1 overexpression groups, $\mathrm{HR}>1$ indicated worse survival. If there was no overlap between $95 \% \mathrm{CI}$ and $\mathrm{HR}=1$, it would be considered that the impact of FOXQ1 on survival was statistically significant. Sensitivity analysis was carried out to validate the credibility of the meta-analysis outcomes by sequentially excluding one study at a time. If the results did not obviously change when one study was removed, the sensitivity is low, and the results are robust. Publication bias was assessed through Begg's test and Egger's test, and $P<0.05$ was recognized as statistically significant. All statistical analyses were conducted using the STATA12.0 software (Stata Corporation, College Station, TX, USA). Ethical approval was not required as this study does not report on or involve the use of any animal or human data or tissue.

\section{Results}

\section{Study selection and characteristics}

The initial search identified 95 relevant studies. Based on the screening of the titles and abstracts of all studies, 85 studies were retrieved according to the inclusion criteria, leaving 10 articles available for full-text review. After carefully reviewing and assessing, four studies were removed due to various reasons. Therefore, six studies containing seven cohorts were finally included in this metaanalysis. ${ }^{13,15,16,19,21,22}$ Figure 1 shows the process of study

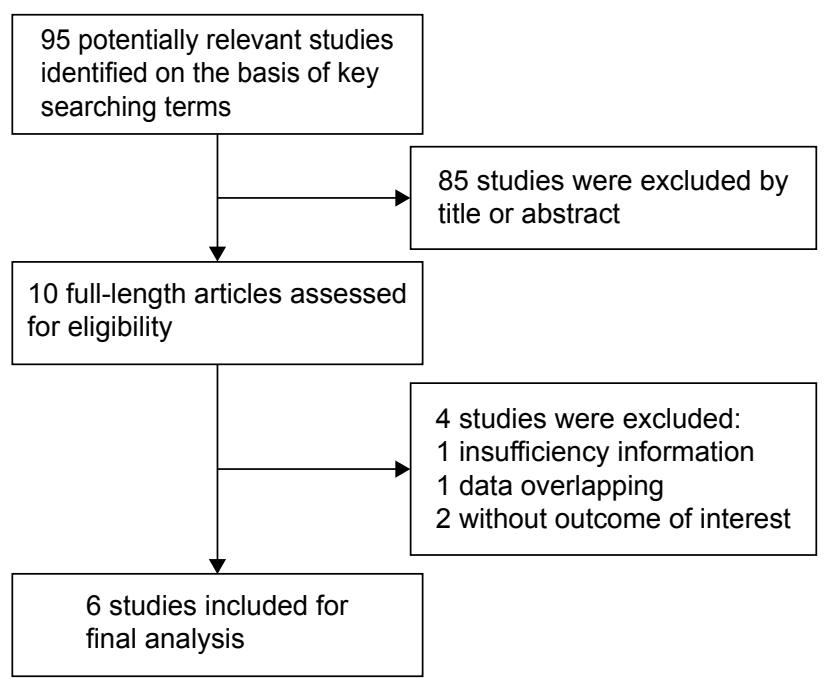

Figure I Selection flowchart of the meta-analysis. 
Table I Main characteristics of all studies included in the meta-analysis

\begin{tabular}{|c|c|c|c|c|c|c|c|}
\hline First author (year) & $\begin{array}{l}\text { Patient } \\
\text { source }\end{array}$ & Histology & $\begin{array}{l}\text { Number } \\
\text { of patients }\end{array}$ & Method & $\begin{array}{l}\text { Positive } \\
\text { (\%) }\end{array}$ & HR estimation & $\begin{array}{l}\text { Survival } \\
\text { results }\end{array}$ \\
\hline Xia et al ${ }^{16}$ Cohort I & China & $\mathrm{HCC}$ & 690 & $\mathrm{IHC}$ & 54.5 & $\mathrm{HR}$ and $95 \% \mathrm{Cl}: 1.27(1.03-1.56)$ & Poor \\
\hline Xia et al ${ }^{16}$ Cohort II & China & $\mathrm{HCC}$ & 312 & $\mathrm{IHC}$ & 53.8 & $\mathrm{HR}$ and $95 \% \mathrm{Cl}$ : I.48 (I.09-2.01) & Poor \\
\hline Zhan et $\mathrm{a}^{21}$ & China & PC & 83 & $\mathrm{IHC}$ & 41.0 & $\mathrm{HR}$ and $95 \% \mathrm{Cl}: 1.856(1.065-3.234)$ & Poor \\
\hline Feng et $\mathrm{al}^{13}$ & China & NSCLC & 103 & $\mathrm{IHC}$ & 79.6 & HR and $95 \% \mathrm{Cl}$ : 2.09I (I.009-4.332) & Poor \\
\hline Liang et $\mathrm{a}^{19}$ & China & GC & 158 & $\mathrm{IHC}$ & 53.8 & $\mathrm{HR}$ and $95 \% \mathrm{Cl}: \mathrm{I} .286(0.746-2.248)$ & Poor \\
\hline Zhang et $\mathrm{a}^{22}$ & China & GC & 60 & $\mathrm{IHC}$ & 63.3 & $\mathrm{HR}$ and $95 \% \mathrm{Cl}: 1.942$ (I.057-3.567) & Poor \\
\hline Wang et $\mathrm{al}^{15}$ & China & $\mathrm{HCC}$ & 114 & $\mathrm{IHC}$ & 68.4 & $\mathrm{HR}$ and $95 \% \mathrm{Cl}$ : 2.69I (I.364-5.309) & Poor \\
\hline
\end{tabular}

Abbreviations: $\mathrm{Cl}$, confidence interval; GC, gastric cancer; HR, hazard ratio; HCC, hepatocellular carcinoma; IHC, immunohistochemistry; NSCLC, non-small-cell lung cancer; PC, pancreatic cancer.

selection for this meta-analysis. The main characteristics of the six included studies containing seven cohorts are extracted and summarized in Table 1. Eventually, a total of 1,520 patients with malignant solid tumors were retained for subsequent meta-analysis. These six studies of good quality were published between 2012 and 2016. All the studies were conducted in China. All the studies examined the prognostic value of FOXQ1 overexpression in patients with solid tumors. Five of the six studies reported HRs (multivariate analysis) explicitly. An HR on OS could be extracted for our original data. All studies identified FOXQ1 overexpression as an indicator of poor OS.

\section{Meta-analysis}

The results of the meta-analysis regarding the correlation between FOXQ1 overexpression and OS are shown in Figure 2. Overall, the combined HR for all six eligible studies (seven cohorts) evaluating FOXQ1 expression and OS indicated that high FOXQ1 expression was associated with poor prognosis of solid tumors for $\mathrm{OS}$ ( $\mathrm{HR}=1.38$; 95\% CI: $1.17-1.59 ; P<0.001$; random effects). No significant heterogeneity was present among all included cohorts $\left(I^{2}=0.0 \%, P=0.568\right)$.

In subgroup analyses (Figure 2), three cohorts related to HCC were divided into the same group, with the combined

\begin{tabular}{|c|c|c|}
\hline Study ID & $\mathrm{HR}(95 \% \mathrm{Cl})$ & Weight (\%) \\
\hline $\mathrm{HCC}$ & & \\
\hline Xia et $a{ }^{16}$ Cohort I & $1.27(1.03-1.56)$ & 62.34 \\
\hline Xia et al ${ }^{16}$ Cohort II & $1.48(1.09-2.01)$ & 20.69 \\
\hline Wang et $\mathrm{al}^{15}$ & $2.69(1.36-5.31)$ & 1.13 \\
\hline Subtotal $\left(I^{2}=17.6 \%, P=0.297\right)$ & $1.34(1.11-1.57)$ & 84.15 \\
\hline Others & & \\
\hline Zhan et $a^{21}$ & $1.86(1.07-3.23)$ & 3.72 \\
\hline Feng et $a^{13}$ & $2.09(1.01-4.33)$ & 1.59 \\
\hline Liang et al ${ }^{19}$ & $1.29(0.75-2.25)$ & 7.76 \\
\hline Zhang et $\mathrm{al}^{22}$ & $1.94(1.06-3.57)$ & 2.78 \\
\hline Subtotal $\left(I^{2}=0.0 \%, P=0.682\right)$ & $1.62(1.09-2.14)$ & 15.85 \\
\hline Heterogeneity between groups: $P=0.347$ & & \\
\hline Overall $\left(I^{2}=0.0 \%, P=0.568\right)$ & $1.38(1.17-1.59)$ & 100 \\
\hline
\end{tabular}

Figure 2 In subgroup analyses, forest plot of HR for the association of FOXQI expression with OS in patients with malignant solid tumors. Abbreviations: $\mathrm{Cl}$, confidence interval; FOXQI, forkhead box QI; HCC, hepatocellular carcinoma; HR, hazard ratio; OS, overall survival. 


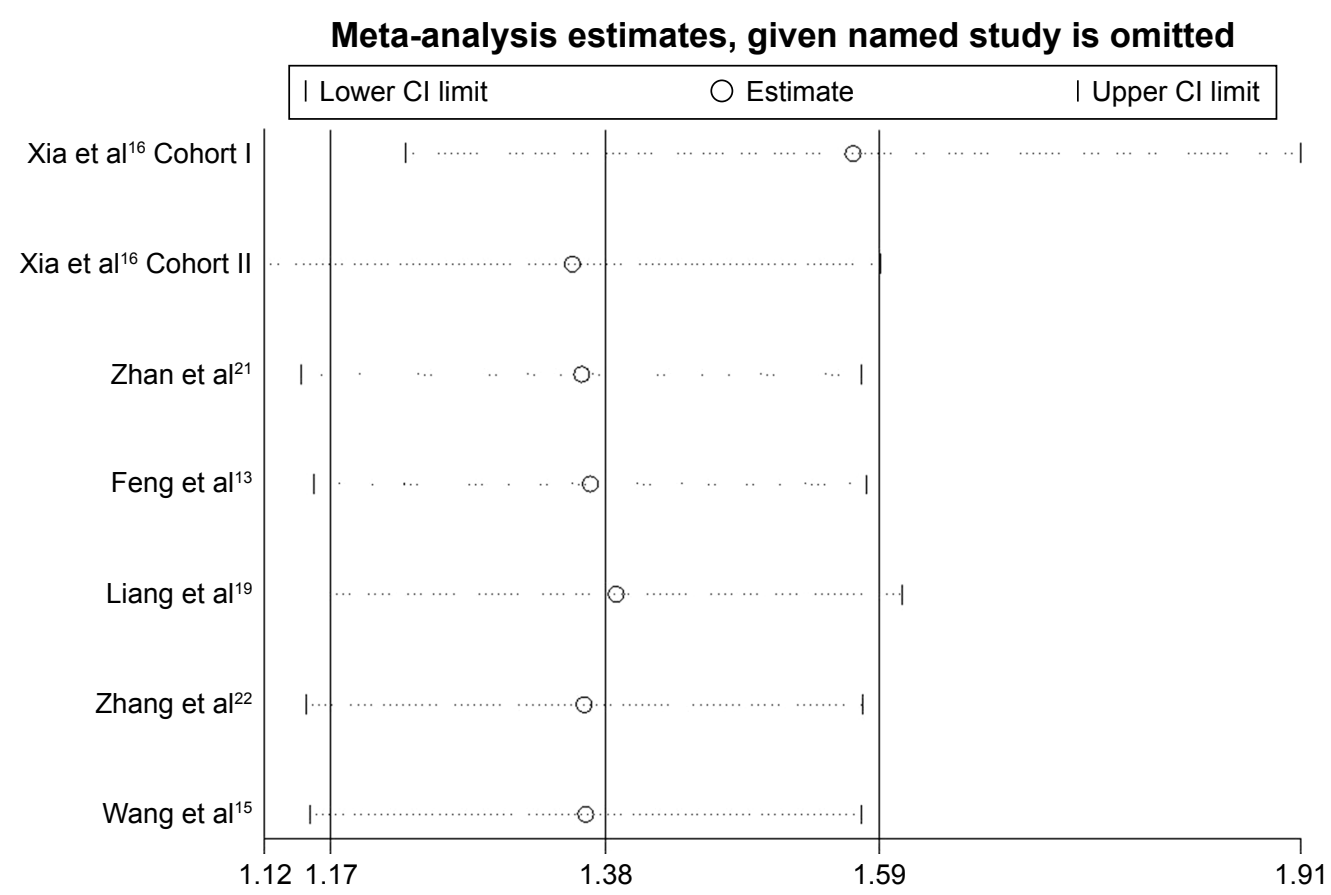

Figure 3 Results of sensitivity analysis by omitting each study at a time.

Notes: Rows represent the results of meta-analysis of all studies except the omitted study named in that row. Omission of any study did not affect the whole estimate results significantly.

Abbreviation: $\mathrm{Cl}$, confidence interval.

$\mathrm{HR}=1.34$ (95\% CI: $1.11-1.57)$. There was a statistical significance between the expression of FOXQ1 and the prognosis of HCC $(P<0.001)$. There was no heterogeneity among various studies $\left(I^{2}=17.6 \%, P=0.297\right)$. Four cohorts related to other solid tumors were divided into others, with the combined HR $=1.62$ (95\% CI: 1.09-2.14). There was a significant statistical significance between the expression of FOXQ1 and the prognosis of others $(P<0.001)$. There was also no heterogeneity among various studies $\left(I^{2}=0.00 \%, P=0.682\right)$.

\section{Sensitivity analysis}

To test the robustness of the HR estimates, sensitivity analysis was performed to evaluate the impact of individually excluding studies on the final result of meta-analysis. As shown in Figure 3 , the corresponding pooled HRs were not significantly altered, suggesting the robustness of our results.

\section{Publication bias}

The interpretability of publication bias assessed by Begg's and Egger's tests was limited when only six studies were included in this meta-analysis.

\section{Discussion}

As a transcription factor, forkhead family protein plays important roles in cell cycle regulation, embryonic development, lipid and carbohydrate metabolism, aging, immune regulation, and other biological processes. ${ }^{4,23,24}$ As a member of forkhead family protein, in mammals, FOXQ1 was initially found to take part in hair follicle development. ${ }^{8}$ Thereafter, it was found to be overexpressed in various cancers. ${ }^{9-19}$ Its upregulation enhances tumor growth and invasion..$^{10,12,14,16}$ Moreover, increases in FOXQ1 are predictive of poor prognosis in patients with various malignant solid tumors. ${ }^{9-19}$ However, thus far, no meta-analyses have been carried to evaluate the prognostic value of increased FOXQ1 in patients with solid tumors. To the best of our knowledge, this is the first comprehensive meta-analysis of the effects of increased FOXQ1 expression on the survival of malignant solid tumors.

In this meta-analysis, we performed a meta-analysis to evaluate the prognostic value of FOXQ1 by combining six eligible studies including 1,520 malignant solid tumor patients. The data were organized according to OS. Combined HRs indicated that high FOXQ1 expression was associated with poor prognosis of OS ( $\mathrm{HR}=1.38$; 95\% CI: 1.17-1.59) in patients with solid tumors. The subgroup analysis revealed that the elevated levels of FOXQ1 appear to be worse OS in HCC (HR $=1.34 ; 95 \%$ CI: $1.11-1.57 ; P<0.001)$ and other cancers (HR $=1.62 ; 95 \%$ CI: 1.09-2.14; $P<0.001$ ).

Publication bias was a problem to be analyzed in all of the meta-analysis. In this study, the interpretability of publication bias assessed by Begg's and Egger's tests was limited when only six studies were included in this meta-analysis; 
so, we did not perform Begg's and Egger's tests. Sensitivity analyses further revealed the robustness of the meta-analysis findings.

Several limitations should be taken into consideration when interpreting the results of our meta-analysis. 1) Only six relevant articles of seven cohorts were only involved with 1,520 patients and all designed in China. 2) The low number of included cohorts limited our evaluation of a potential publication bias. Therefore, publication bias may exist. 3) The method used for the assessment of FOXQ1 expression is IHC, and experimental IHC protocols could have confounded the results, and the cutoff definition of FOXQ1 overexpression may led to between-study heterogeneity.

\section{Conclusion}

Our study indicated that overexpression of FOXQ1 may lead to poor prognosis. With the gradual deepening of the study, FOXQ1 may become an important target for cancer therapy in the future.

\section{Acknowledgments}

We thank Li Wang, Jiansheng Wang, and Yunfeng Zhang for the design and acquisition of data of this study. The authors wish to share any specific data upon request. As of now, there are no additional data, other than those reported in this article.

\section{Disclosure}

The authors report no conflicts of interest in this work.

\section{References}

1. Myatt SS, Lam EW. The emerging roles of forkhead box (Fox) proteins in cancer. Nat Rev Cancer. 2007;7(11):847-859.

2. Katoh M, Katoh M. Human FOX gene family (Review). Int J Oncol. 2014; 25(5):1495-1500.

3. Feuerborn A, Srivastava PK, Kuffer S, et al. The Forkhead factor FoxQ1 influences epithelial differentiation. J Cell Physiol. 2011;226(3): 710-719.

4. Eijkelenboom A, Burgering BM. FOXOs: signalling integrators for homeostasis maintenance. Nat Rev Mol Cell Biol. 2013;14(2):83-97.

5. Jonsson H, Peng SL. Forkhead transcription factors in immunology. Cell Mol Life Sci. 2005;62(4):397-409.

6. Goering W, Adham IM, Pasche B, et al. Impairment of gastric acid secretion and increase of embryonic lethality in Foxq1-deficient mice. Cytogenet Genome Res. 2008;121(2):88-95.
7. Hong HK, Noveroske JK, Headon DJ, et al. The winged helix/forkhead transcription factor Foxq1 regulates differentiation of hair in satin mice. Genesis. 2001;29(4):163-171.

8. Potter CS, Peterson RL, Barth JL, et al. Evidence that the satin hair mutant gene Foxq1 is among multiple and functionally diverse regulatory targets for Hoxc13 during hair follicle differentiation. J Biol Chem. 2006;281(39):29245-29255.

9. Qiao Y, Jiang X, Lee ST, Karuturi RK, Hooi SC, Yu Q. FOXQ1 regulates epithelial-mesenchymal transition in human cancers. Cancer Res. 2011;71(8):3076-3086.

10. Zhang H, Meng F, Liu G, et al. Forkhead transcription factor foxq1 promotes epithelial-mesenchymal transition and breast cancer metastasis. Cancer Res. 2011;71(4):1292-1301.

11. Sehrawat A, Kim SH, Vogt A, Singh SV. Suppression of FOXQ1 in benzyl isothiocyanate-mediated inhibition of epithelial-mesenchymal transition in human breast cancer cells. Carcinogenesis. 2013;34(4): 864-873.

12. Kaneda H, Arao T, Tanaka K, et al. FOXQ1 is overexpressed in colorectal cancer and enhances tumorigenicity and tumor growth. Cancer Res. 2010;70(5):2053-2063.

13. Feng J, Zhang X, Zhu H, Wang X, Ni S, Huang J. FoxQ1 overexpression influences poor prognosis in non-small cell lung cancer, associates with the phenomenon of EMT. PLoS One. 2012;7(6):e39937.

14. Feng J, Xu L, Ni S, et al. Involvement of FoxQ1 in NSCLC through regulating EMT and increasing chemosensitivity. Oncotarget. 2014; 5(20):9689-9702.

15. Wang W, He S, Ji J, Huang J, Zhang S, Zhang Y. The prognostic significance of FOXQ1 oncogene overexpression in human hepatocellular carcinoma. Pathol Res Pract. 2013;209(6):353-358.

16. Xia L, Huang W, Tian D, et al. Forkhead box Q1 promotes hepatocellular carcinoma metastasis by transactivating ZEB2 and VersicanV1 expression. Hepatology. 2014;59(3):958-973.

17. Pei Y, Wang P, Liu H, He F, Ming L. FOXQ1 promotes esophageal cancer proliferation and metastasis by negatively modulating $\mathrm{CDH} 1$ Biomed Pharmacother. 2015;74:89-94.

18. Peng X, Luo Z, Kang Q, et al. FOXQ1 mediates the crosstalk between TGF-beta and Wnt signaling pathways in the progression of colorectal cancer. Cancer Biol Ther. 2015;16(7):1099-1109.

19. Liang SH, Yan XZ, Wang BL, et al. Increased expression of FOXQ1 is a prognostic marker for patients with gastric cancer. Tumour Biol. 2013; 34(5):2605-2609.

20. Stang A. Critical evaluation of the Newcastle-Ottawa scale for the assessment of the quality of nonrandomized studies in meta-analyses. Eur J Epidemiol. 2010;25(9):603-605.

21. Zhan HX, Xu JW, Wang L, Wu D, Zhang GY, Hu SY. FoxQ1 is a novel molecular target for pancreatic cancer and is associated with poor prognosis. Curr Mol Med. 2015;15(5):469-477.

22. Zhang J, Liu Y, Zhang J, et al. FOXQ1 promotes gastric cancer metastasis through upregulation of Snail. Oncol Rep. 2016;35(6):3607-3613.

23. Oellerich MF, Potente M. FOXOs and sirtuins in vascular growth, maintenance, and aging. Circ Res. 2012;110(9):1238-1251.

24. Wen Q, Wang H, Little PJ, Quirion R, Zheng W. Forkhead family transcription factor FoxO and neural differentiation. Neurogenetics. 2012; 13(2):105-113.
OncoTargets and Therapy

\section{Publish your work in this journal}

OncoTargets and Therapy is an international, peer-reviewed, open access journal focusing on the pathological basis of all cancers, potential targets for therapy and treatment protocols employed to improve the management of cancer patients. The journal also focuses on the impact of management programs and new therapeutic agents and protocols on

\section{Dovepress}

patient perspectives such as quality of life, adherence and satisfaction. The manuscript management system is completely online and includes a very quick and fair peer-review system, which is all easy to use. Visit http://www.dovepress.com/testimonials.php to read real quotes from published authors. 